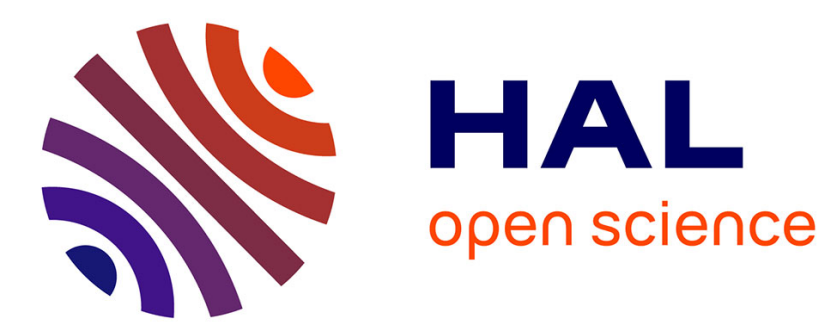

\title{
Experimental and analytical study of the shear instability of a gas-liquid mixing layer
}

Jean-Philippe Matas, Sylvain Marty, Alain H. Cartellier

\section{To cite this version:}

Jean-Philippe Matas, Sylvain Marty, Alain H. Cartellier. Experimental and analytical study of the shear instability of a gas-liquid mixing layer. Physics of Fluids, 2011, 23, pp.094112. 10.1063/1.3642640 . hal-00644002

\section{HAL Id: hal-00644002 https://hal.science/hal-00644002}

Submitted on 3 Jan 2012

HAL is a multi-disciplinary open access archive for the deposit and dissemination of scientific research documents, whether they are published or not. The documents may come from teaching and research institutions in France or abroad, or from public or private research centers.
L'archive ouverte pluridisciplinaire HAL, est destinée au dépôt et à la diffusion de documents scientifiques de niveau recherche, publiés ou non, émanant des établissements d'enseignement et de recherche français ou étrangers, des laboratoires publics ou privés. 


\section{Experimental and analytical study of the shear instability of a gas- liquid mixing layer}

Jean-Philippe Matas, Sylvain Marty, and Alain Cartellier

Citation: Phys. Fluids 23, 094112 (2011); doi: 10.1063/1.3642640

View online: http://dx.doi.org/10.1063/1.3642640

View Table of Contents: http://pof.aip.org/resource/1/PHFLE6/v23/i9

Published by the American Institute of Physics.

\section{Related Articles}

Modal versus nonmodal linear stability analysis of river dunes

Phys. Fluids 23, 104102 (2011)

On the transition between distributed and isolated surface roughness and its effect on the stability of channel flow

Phys. Fluids 23, 104101 (2011)

Optimal excitation of two dimensional Holmboe instabilities

Phys. Fluids 23, 074102 (2011)

Coupling of Kelvin-Helmholtz instability and buoyancy instability in a thermally laminar plasma

Phys. Plasmas 18, 022110 (2011)

Competitions between Rayleigh-Taylor instability and Kelvin-Helmholtz instability with continuous density and velocity profiles

Phys. Plasmas 18, 022704 (2011)

\section{Additional information on Phys. Fluids}

Journal Homepage: http://pof.aip.org/

Journal Information: http://pof.aip.org/about/about_the_journal

Top downloads: http://pof.aip.org/features/most_downloaded

Information for Authors: http://pof.aip.org/authors

\section{ADVERTISEMENT}

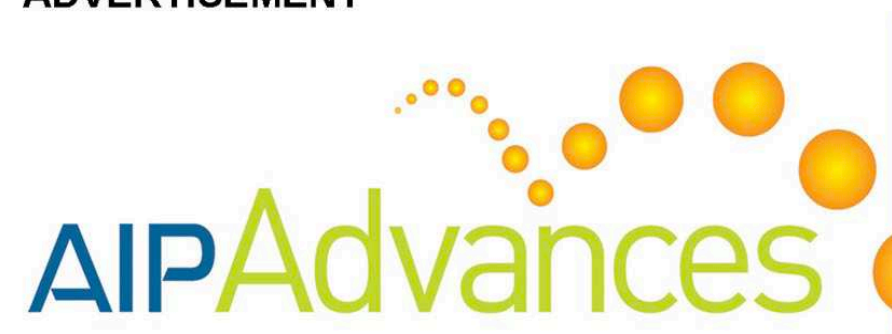

Submit Now

Explore AIP's new

open-access journal

- Article-level metrics now available

Join the conversation! Rate $\&$ comment on articles 


\title{
Experimental and analytical study of the shear instability of a gas-liquid mixing layer
}

\author{
Jean-Philippe Matas, ${ }^{\text {a) }}$ Sylvain Marty, and Alain Cartellier \\ Laboratoire des Ecoulements Géophysiques et Industriels, CNRS, Grenoble University, \\ 38041 Grenoble Cedex 9, France
}

(Received 23 September 2010; accepted 16 August 2011; published online 29 September 2011)

\begin{abstract}
We carry out an inviscid spatial linear stability analysis of a planar mixing layer, where a fast gas stream destabilizes a slower parallel liquid stream, and compare the predictions of this analysis with experimental results. We study how the value of the liquid velocity at the interface and the finite thickness of the gas jet affect the most unstable mode predicted by the inviscid analysis: in particular a zero interface velocity is considered to account for the presence in most experimental situations of a splitter splate separating the gas and the liquid. Results derived from this theory are compared with experimentally measured frequencies and growth rates: a good agreement is found between the experimental and predicted frequencies, while the experimental growth rates turn out to be much larger than expected. (C) 2011 American Institute of Physics. [doi:10.1063/1.3642640]
\end{abstract}

\section{INTRODUCTION}

Airblast atomization is used to turn a liquid jet into a spray with a fast cocurrent air stream. This process is exploited in particular in aeronautics and space applications for the injectors of several propulsion systems. ${ }^{1}$ The mechanisms responsible for the break-up of the liquid jet are only partially understood, see reviews by Lasheras and Hopfinger, ${ }^{2}$ and Eggers and Villermaux. ${ }^{3}$ However, an improvement of injection techniques is needed to notably limit the emission of pollutants and to increase the reliability of these engines.

It has been shown that the initial destabilization of the interface in airblast atomization is caused by a shear instability akin to a Kelvin-Helmholtz instability, but whose most unstable mode is controlled by the thickness $\delta_{g}$ of the gas vorticity layer. See for example the original work by Rayleigh ${ }^{4}$ with the inclusion of a velocity profile in the shear instability, or the work of Lawrence et al. ${ }^{5}$ where both a velocity and density profile are taken into account. Several recent studies ${ }^{6-9}$ have focused on the case of gas-liquid shear layers, relevant to atomization applications. When a stability analysis is carried out with both a gas and a liquid boundary layer (see velocity profile of Fig. 1(a)), the most unstable mode turns out to be directly controlled by the liquid vorticity layer $\delta_{l}$, and not the gas one. ${ }^{10,11}$ This is not what is observed in the experiments of Raynal, ${ }^{7,12}$ Marmottant and Villermaux, ${ }^{8}$ and Ben Rayana. ${ }^{9,13}$ This inconsistency has been resolved by pointing out that the time needed for the liquid shear layer to grow by viscous diffusion is large compared with the time needed for the gas mode to grow. ${ }^{3,8,12}$

Under the assumption that there is a vorticity layer only on the gas side (see Fig. 1(b)), an explicit expression for the dispersion relation is then found; in the limit of large Weber and small Richardson numbers the wavenumber of its most unstable temporal solution is found numerically to scale $\mathrm{as}^{3}$ :

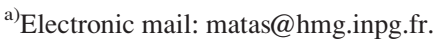

$$
k \approx 1.5\left(\frac{\rho_{l}}{\rho_{g}}\right)^{1 / 2} \frac{1}{\delta_{g}}
$$

The velocity of the waves can be well approximated by the following convection velocity, estimated from a pressure balance between the liquid and gas in a frame moving with the waves ${ }^{7,12,14}$ :

$$
U_{c}=\frac{\sqrt{\rho_{l}} U_{l}+\sqrt{\rho_{g}} U_{g}}{\sqrt{\rho_{g}}+\sqrt{\rho_{l}}}
$$

The frequency of the waves can then be approximated by $f=U_{c} / \lambda$, with $\lambda$ is the wavelength. Experimental results show wavelengths scaling with $\delta_{g}$ and frequencies scaling as $U_{c} / \delta_{g}$ as predicted above, ${ }^{6-8,12,13}$ but measured frequency values are consistently larger than predicted. There is also a disagreement between existing experimental data: Raynal $e t a l .^{7,12}$ find experimental frequencies about 50\% larger than predicted ones, whereas Ben Rayana et $a l^{9,13}$ on the same planar geometry and Marmottant and Villermaux ${ }^{8}$ on a coaxial geometry measured a factor two or three between the theory and experiments.

If viscosity is taken into account in the temporal analysis, an additional unstable mode is found, ${ }^{15,16}$ with a much shorter wavelength than both the inviscid and the experimental modes. For density ratios corresponding to air/water experiments Boeck and Zaleski ${ }^{15}$ find that this viscous mode is the most unstable one. However, contrary to the inviscid and the experimental modes, this mode does not scale with the gas vorticity thickness $\delta_{g}$ : we therefore choose not to include viscosity in our stability analysis, and will focus instead on the improvement of the inviscid analysis. We will discuss later on what our results suggest as to how viscosity may be included in the analysis.

In order to clarify the discrepancies between the inviscid stability analysis and experimental results, we have extended the analysis to integrate key aspects of the injection: a more realistic initial velocity profile accounting for the necessary presence of a splitter plate in the experiments, and the finite 


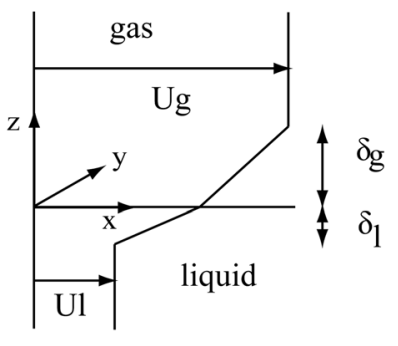

(a)

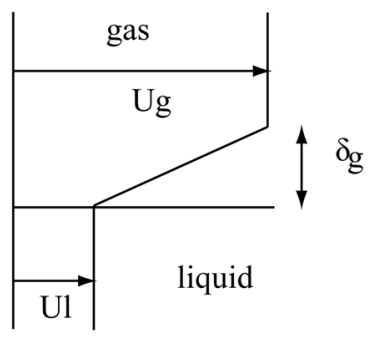

(b)

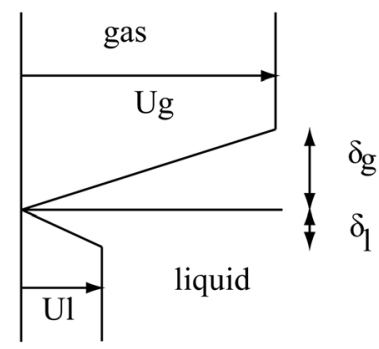

(c)
FIG. 1. (a) Velocity profile with two vorticity thicknesses; (b) Velocity profile with a gas vorticity thickness only; and (c) Profile exhibiting a velocity deficit at the interface. thickness of the gas stream. We will show in Sec. II that these changes significantly modify the predictions of the inviscid stability analysis. We will then compare in Sec. III these predictions with both existing and new experimental results.

\section{INVISCID ANALYSIS}

The stability analysis is carried along the lines of the inviscid analysis carried out by Raynal et al. ${ }^{7}$ and Raynal. ${ }^{12}$ We note $\mathrm{U}$ the base flow, and $\mathbf{u}=(u, v, w)$ the velocity perturbation. The position of the interface is given by $\eta$. The pressure perturbation is $p$. We note $U_{i}$ the base flow velocity at the interface. The influence of the surface tension $\sigma$ and of gravity will be neglected in this section.

We linearize the classical equations of momentum and mass conservation, and look for normal mode solutions $\tilde{\mathbf{u}}\left(k_{x}, k_{y}, z, \omega\right) e^{i\left(k_{x} x+k_{y} y-\omega t\right)}$ of the resulting system. Solving for the vertical component of the perturbation velocity $\tilde{w}$, we obtain the following equation ${ }^{17}$ :

$$
\frac{d}{d z}\left[\rho\left(-\omega+k_{x} U\right) \frac{d \tilde{w}}{d z}-\rho k_{x} \frac{d U}{d z} \tilde{w}\right]-\rho k^{2}\left(-\omega+k_{x} U\right) \tilde{w}=0
$$

where $k^{2}=k_{x}^{2}+k_{y}^{2}$. Away from the interface, $\rho$ is constant and this equation reduces to:

$$
\rho\left(-\omega+k_{x} U\right)\left(\frac{d^{2} \tilde{w}}{d z^{2}}-k^{2} \tilde{w}\right)=\rho k_{x} \frac{d^{2} U}{d z^{2}} \tilde{w}
$$

As discussed in the introduction, the nature of the solution for $\tilde{w}$ depends on the velocity profile chosen for $U(z)$. If a linear profile is adopted, then the term on the right hand side of Eq. (4) is zero: an analytical solution can easily be provided for $\tilde{w}$, and with proper jump conditions at the interface yield an expression for the dispersion relation.

\section{A. Effect of a velocity deficit}

We have studied the stability of the base flow showed on Fig. 1(c). This base flow typically exists just at the exit of the injector in the experimental set-up, due to the solid plate separating the liquid and gas flows. Viscosity will lead to a diffusion of momentum towards the interface, and the profile of Fig. 1(c) is expected to hold only over a short distance, and evolve eventually towards the profile of Fig. 1(a). However, a velocity deficit, meaning a velocity close to the inter- face lower than the bulk liquid velocity, has been observed in our experimental set-up with particle image velocimetry (PIV) measurements (seeding with rhodamine and Nd-YAG laser slice). Only a couple of velocity vectors could be obtained within the liquid boundary layer, so its full velocity profile could not be measured, but we could check that even in the presence of the fast gas flow the liquid velocity remained smaller in the liquid boundary layer than in the bulk of the liquid, up to several centimeters downstream the injection.

An error function velocity profile can be used in the analysis instead of the linear velocity profiles of Fig. 1: however, this more realistic profile yields exactly the same predicted unstable modes for our inviscid case, provided the gas vorticity thickness $\delta_{g}=\Delta U /\left.\frac{d U}{d z}\right|_{\max }$ is the same in both cases. We therefore choose to present here results obtained with the more straightforward linear velocity profile. Note that though viscosity is not included in the derivation of the stability of the velocity perturbation, it is of course implicitly needed upstream to generate the base flow profiles of Fig. 1.

For all three base flows of Fig. 1, Eq. (4) then reduces to

$$
\frac{d^{2} \tilde{w}}{d z^{2}}-k^{2} \tilde{w}=0
$$

Two solutions are built in the gas phase: $w_{1}=A e^{-k z}$ in the unbounded constant velocity region and $w_{2}=B e^{-k z}+C e^{k z}$ in the boundary layer; similarly two solutions are built in the liquid phase: $w_{3}=D e^{-k z}+E e^{k z}$ and $w_{4}=F e^{k z}$. These solutions are built under the assumption $\Re(k)>0$ (waves propagating downstream). Continuity of $w$ at the limit between constant flow and linear flow zones in each phase, and across the interface, i.e., at $\mathrm{z}=-\delta_{g}, 0$ and $\delta_{g}$, gives three relations between the six integration constants. Three additional jump conditions are obtained by integrating Eq. (3) across the same three locations (i.e. across corner points of the velocity profile) ${ }^{17}$ :

$$
\begin{aligned}
\left(-\omega+k_{x} U_{g}\right) k\left[A e^{-k \delta_{g}}-B e^{-k \delta_{g}}+C k e^{k \delta_{g}}\right]-k_{x} \gamma_{g} A e^{-k \delta_{g}} & =0 \\
\left(-\omega+k_{x} U_{l}\right) k\left[-D e^{-k \delta_{l}}+E k e^{k \delta_{l}}-F e^{k \delta_{l}}\right]-k_{x} \gamma_{l} F e^{k \delta_{l}} & =0 \\
\left(-\omega+k_{x} U_{i}\right) k\left[\rho_{g}(-B+C)-\rho_{l}(-D+E)\right] & \\
-\rho_{g} k_{x} \gamma_{g}(B+C)+\rho_{l} k_{x} \gamma_{l}(D+E) & =0
\end{aligned}
$$

where $\gamma_{g}=\left(U_{g}-U_{i}\right) / \delta_{g}$ and $\gamma_{l}=\left(U_{i}-U_{l}\right) / \delta_{l}$. We have taken $k_{y}=0$, meaning that we look for modes propagating in the $x$ direction. The dispersion relation is obtained by writing that the determinant of this system must be zero: 


$$
\begin{array}{|cccccc}
0 & 1 & 1 & -1 & -1 \\
1 & -1 & -e^{2 K} & 0 & 0 & 0 \\
0 & 0 & 0 & -e^{2 K / n} & -1 & 1 \\
0 & {\left[-r-\frac{r\left(1-\alpha_{i}\right)+n\left(\alpha_{l}-\alpha_{i}\right)}{-\Omega+K \alpha_{i}}\right]} & {\left[r-\frac{r\left(1-\alpha_{i}\right)+n\left(\alpha_{l}-\alpha_{i}\right)}{-\Omega+K \alpha_{i}}\right]} & 0 \\
-1+\frac{1-\alpha_{i}}{-\Omega+K} & 1 & -e^{2 K} & 0 & 0 & 0 \\
0 & 0 & 0 & -e^{2 K / n} & 1 & -1-n \frac{\alpha_{i}-\alpha_{l}}{-\Omega+K \alpha_{l}}
\end{array} \mid=0
$$

where $r=\rho_{g} / \rho_{l}$ is the density ratio, $n$ is the vorticity thickness ratio $n=\delta_{g} / \delta_{l}, \alpha_{i}=U_{i} / U_{g}$, and $\alpha_{l}=U_{l} / U_{g}$ are the interface and liquid velocities nondimensionalized by $U_{g}$. The frequency and wavenumber are made nondimensional by setting $\Omega=\omega \delta_{g} / U_{g}$ and $K=k \delta_{g}$.

This equation is solved numerically for spatial solutions: the variable is the real $\omega$, and the dispersion relation is solved for the complex wavenumber $k=k_{r}+i k_{i}$. The frequency retained is the one which minimizes the (negative) spatial growth rate $k_{i}$. Note that with the form of the perturbation taken above, the perturbation is unstable if $k_{i}<0$. Figure 2 shows typical variations of $k_{r}$ and $k_{i}$, as a function of nondimensionalized frequency $\omega \delta_{g} / U_{g}$. The dotted curves represent the curves obtained with the velocity profiles of Fig. 1(b) (corresponding to $\alpha_{i}=\alpha_{l}$ ), while the solid curve is obtained with a velocity profile exhibiting a velocity deficit at the interface $\left(\alpha_{i}=0\right.$, Fig. 1(c)). It can be seen that for the conditions of Fig. $2\left(r=10^{-3}, \alpha_{l}=10^{-2}\right.$, and $\left.n=1\right)$ the unstable mode obtained with a velocity deficit has a larger frequency. The absolute value of the (negative) growth rate is also slightly larger. Note also that when the velocity deficit is taken into account the unstable mode scaling with $\delta_{l}$, which would be dominant ${ }^{3}$ if the analysis were carried out with the profile of Fig. 1(a), is not observed: a large viscous diffusion time therefore need not be invoked anymore to justify that the most unstable mode scales with $\delta_{g}$.

We plot on Fig. 3 the influence of the velocity ratio $\alpha_{l}$ on the spatial wavenumber and growth rate. The interface velocity is taken to be zero $\left(\alpha_{i}=0\right)$. The dimensionless frequency of the most unstable mode increases with increasing $\alpha_{l}$. It can be seen that when $\alpha_{l}$ is varied in the range $5.10^{-4}-4.10^{-2}$ the

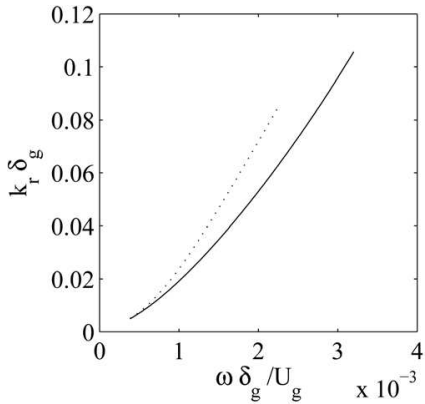

(a)

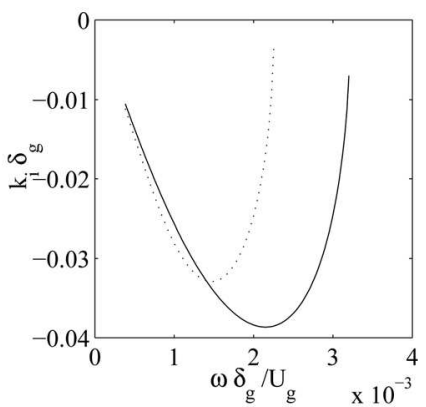

(b)
FIG. 2. (a) Dimensionless wavenumber $k_{r}$ and (b) growth rate $k_{i}$ obtained by stability analysis, as a function of dimensionless frequency $\omega \delta_{g} / U_{g}$. The solid curve is obtained with a base flow having a full velocity deficit at the interface $\left(\alpha_{i}=0\right)$, and the dotted curve with an interface velocity equal to the liquid velocity $\left(\alpha_{i}=\alpha_{l}\right)$. Density ratio $r=10^{-3}$, velocity ratio $\alpha_{l}=10^{-2}$ and vorticity thickness ratio $n=1$. growth rate increases, while for larger $\alpha_{l}$ an increase in $U_{l}$ causes a decrease in the growth rate. Figure 4 compares the variations of the frequency and growth rate of the most unstable mode as a function of the velocity ratio $\alpha_{l}$ in the case where there is a velocity deficit (solid curve, $\alpha_{i}=0$ ) and when the interface velocity is equal to $U_{l}$ (dotted line, $\alpha_{i}=\alpha_{l}$ ). The difference between the two configurations increases with increasing $\alpha_{l}$, up to a factor three in the frequency when $\alpha_{l}$ becomes close to 0.1 . The variations of the growth rate show that for low $\alpha_{l}$ the velocity deficit enhances the instability, while for $\alpha_{l}>5.10^{-3}$ it lowers the magnitude of the growth rate compared with the configuration without a deficit. When the velocity deficit is progressively reduced, i.e., $0<U_{i}<U_{l}$, the results obtained without any liquid boundary layer (Fig. 1(b)) are continuously recovered. If a gravity field perpendicular to the interface is included, it will damp large wavelength modes and therefore increase the most unstable frequency. For the conditions of Fig. 4, which are typical of the experiments presented in Sec. III, the frequency would typically be increased by about $10 \%$. If surface tension is included it will on the contrary decrease slightly the most unstable frequency (by about $2 \%$ for the same typical conditions).

We now plot on Fig. 5 the results of the stability analysis when the ratio $n$ of the gas to liquid vorticity layer is varied in the range $0.15-10$. It can be seen that when $n$ is larger than 0.5 , the frequency, growth rate and velocity of the most unstable mode are roughly independent of $n$. When $n$ is decreased below 0.5 , i.e., when the liquid vorticity layer becomes more than twice as large as the gas vorticity layer, the growth rate and wavenumber are significantly increased, while the group velocity decreases significantly (steeper slope of the wavenumber curve). A consequence of this

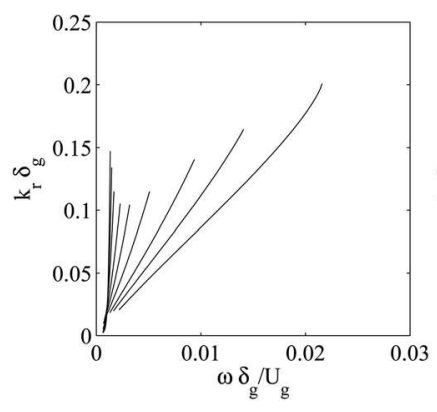

(a)

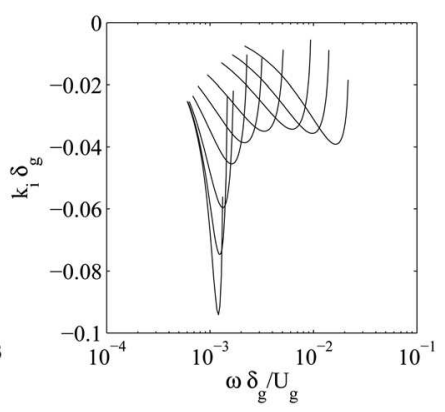

(b)
FIG. 3. Dimensionless wavenumber $k_{r}$ and growth rate $k_{i}$ obtained by stability analysis, as a function of dimensionless frequency $\omega \delta_{g} / U_{g}$, for ratios $U_{l} / U_{g}=5.10^{-4}, 10^{-3}, 2.10^{-3}, 5.10^{-3}, 10^{-2}, 2.10^{-2}, 4.10^{-2}, 6.10^{-2}$, and $9.10^{-2}$ (from left to right). $\alpha_{i}=0, n=1$, and $r=10^{-3}$. 


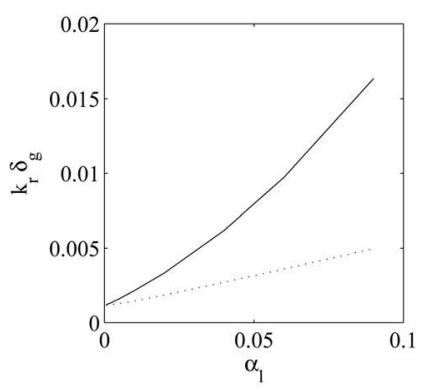

(a)

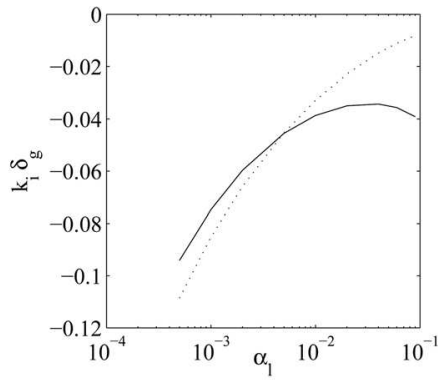

(b)
FIG. 4. (a) Variations of the dimensionless frequency and (b) growth rate of the most unstable mode as a function of the liquid to gas velocity ratio $\alpha_{l}$. Solid curve: velocity deficit at the interface; dotted curve: no velocity deficit. The vorticity thickness ratio $n=\delta_{g} / \delta_{l}$ is fixed at $n=1$, and the density ratio at $r=10^{-3}$.

result is that since experimental conditions on our set-up correspond to situations where $\delta_{g}>\delta_{l}$, the stability analysis predicts that for these conditions the thickness $\delta_{l}$ of the liquid vorticity layer will not affect the nature of the unstable mode when there is a velocity deficit.

\section{B. Asymptotic analysis and influence of the liquid on the gas mode}

Motivated by the fact that previous studies found a relatively simple dependence of the most unstable mode wavelength upon flow parameters in the case without a velocity deficit (see Eq. (1)), and in order to find how this scaling is modified when there is a velocity deficit, we now carry out an asymptotic analysis on the dispersion relation, around the unstable mode identified in Sec. II A. We first make this expansion for the case where there is a single vorticity layer in the gas phase (base velocity profile of Fig. 1(b). We make several assumptions:

- We assume that $r \ll 1$. This is true in the air/water case, but will also hold in any gas/liquid case at moderate absolute pressures. Only lower order terms in $r$ will therefore be retained in the expansion.

- We assume $U_{l} \ll U_{g}$ : this is true in configurations where Kelvin-Helmholtz instability is observed in air/water conditions. In order to include this assumption in the expan-

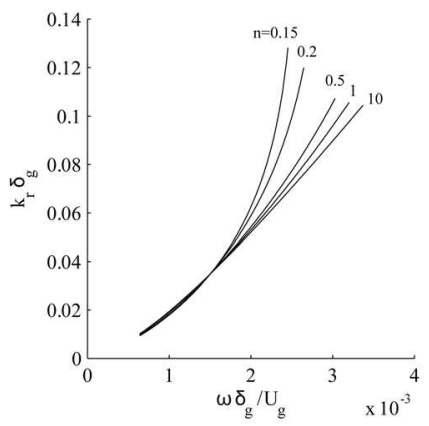

(a)

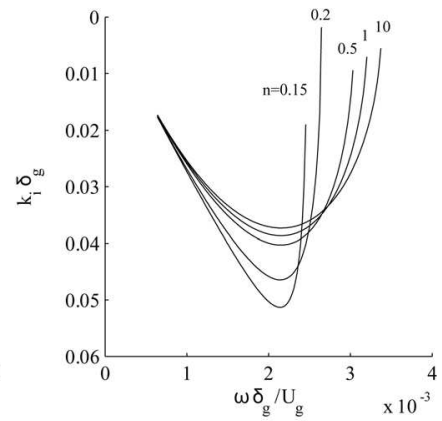

(b)
FIG. 5. (a) Dimensionless wavenumber $k_{r}$ and (b) growth rate $k_{i}$ as a function of dimensionless frequency $\omega \delta_{g} / U_{g}$, for gas to liquid vorticity thickness ratio $n=\delta_{g} / \delta_{l}=0.15,0.2,0.5,1$, and 10 (from left to right). The liquid to gas velocity ratio is fixed $\alpha_{l}=10^{-2}$ and the density ratio $r=10^{-3}$. sion in powers of $r$, we introduce the dynamic pressure ratio $M=\rho_{g} U_{g}^{2} /\left(\rho_{l} U_{l}^{2}\right)$, and assume $\mathrm{M}$ to be of order 1 or larger. We then set $U_{l}=U_{g} \sqrt{r / M}$ in the dispersion relation.

- Based on the scaling of Eq. (1) for the wavenumber, we look for a dimensionless wavenumber $K$ of the form $K=K^{\prime} \sqrt{r}$, where $K^{\prime}=O(1)$. Given the scaling of the phenomenological convection velocity (equation 2 ), we therefore introduce $\Omega=\Omega^{\prime} r$ with $\Omega^{\prime}=O(1)$ as well.

Only the lower order terms in $r$ are kept in the dispersion relation, yielding the simplified dispersion relation:

$$
\Omega^{\prime 2}-\left(K^{\prime 2}+2 \frac{K^{\prime}}{\sqrt{M}}\right) \Omega^{\prime}+\frac{K^{\prime 3}}{\sqrt{M}}+\frac{K^{\prime 2}}{M}+K^{\prime 2}=0
$$

We solve this equation for temporal solutions and find $\Omega^{\prime}=\left(K^{\prime} / 2\right)\left(K^{\prime}+2 / M \pm \sqrt{K^{\prime 2}-4}\right)$. An unstable solution will appear for $K^{\prime}<2$. The most unstable solution when $K^{\prime}$ is varied is found for $K^{\prime}=\sqrt{2}$, corresponding to $\Omega^{\prime}=(1+\sqrt{2 / M}+i)$. The most unstable mode dimensional wavenumber, growth rate, frequency, phase velocity $v_{\varphi}$, and group velocity $v_{g}$ are therefore characterized by:

- $k=\sqrt{2} \frac{\sqrt{r}}{\delta_{g}}$

- $\omega_{i}=r \frac{U_{g}}{\delta_{g}}$ and $\omega_{r}=r \frac{U_{g}}{\delta_{g}}\left(1+\sqrt{2} M^{-1 / 2}\right)$

- $v_{\phi}=\frac{\sqrt{2}}{2} \sqrt{r} U_{g}+U_{l}$ and $v_{g}=\sqrt{2} \sqrt{r} U_{g}+U_{l}$

These expressions agree extremely well with the results found when the complete dispersion relation is solved numerically. They are also consistent with the coefficients found numerically in previous studies (Eq. (1)). The phase velocity is slightly smaller than the convective velocity of Eq. (2). A new and interesting result is the correction in $M$ to the frequency of the most unstable mode: it analytically predicts how the liquid velocity will affect this mode.

We now apply the same method to the case of Fig. 1(c), when there is a velocity deficit at the interface. The same assumptions are made on the magnitude of the liquid

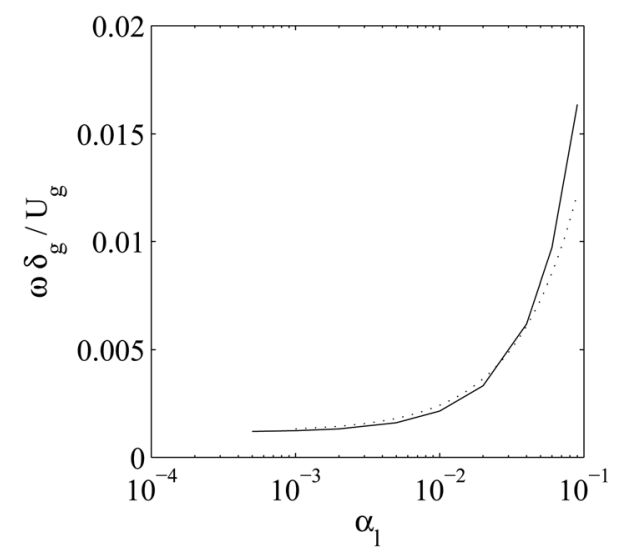

FIG. 6. Frequency of the most unstable mode found when the full dispersion relation is solved numerically for spatial solutions (solid line), and predicted by the asymptotic expansion $\omega_{r}=r \frac{U_{g}}{\delta_{g}}\left(1+\frac{5}{2} \sqrt{2} M^{-1 / 2}\right)$ (dotted line), when the velocity ratio $\alpha_{l}$ is varied. The base velocity profile is taken to have a full velocity deficit at the interface $\left(\alpha_{i}=0\right)$. 


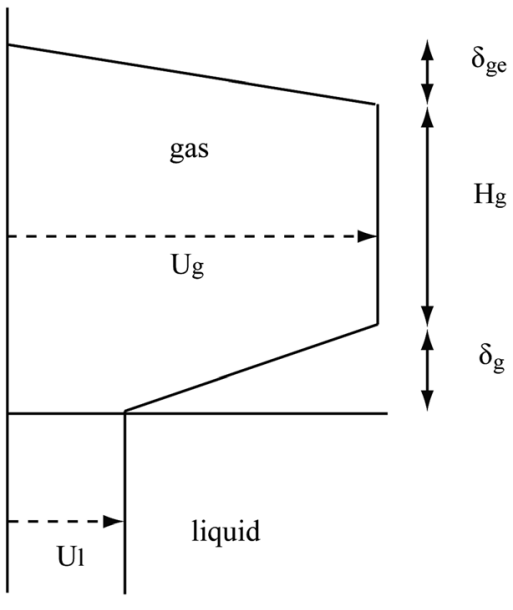

FIG. 7. Velocity profile used for the analysis: the gas jet has a finite thickness $H_{g}$.

velocity, and on the dimensional frequency and wavenumber. In addition, the liquid vorticity thickness has to be such that $K / n \ll 1$, which is equivalent to $k \delta_{l} \ll 1$ : the liquid vorticity thickness has to be small compared with the wavelength of the instability. These approximations lead to the following simplified dispersion relation:

$\Omega^{\prime 3}+\left(\frac{2 K^{\prime}}{\sqrt{M}}+K^{\prime 2}\right) \Omega^{\prime 2}+\left(\frac{2 K^{\prime 3}}{\sqrt{M}}+\frac{K^{\prime 2}}{M}+K^{\prime 2}\right) \Omega^{\prime}+\frac{K^{\prime 4}}{M}=0$

We solve it for temporal solutions in the limit of large $M$ and find:

- $k=\left(\sqrt{2}+\frac{3}{2} M^{-1 / 2}\right) \frac{\sqrt{r}}{\delta_{g}}$

- $\omega_{i}=r \frac{U_{g}}{\delta_{g}}\left(1+\sqrt{2} M^{-1 / 2}\right)$ and $\omega_{r}=r \frac{U_{g}}{\delta_{g}}\left(1+\frac{5}{2} \sqrt{2} M^{-1 / 2}\right)$

- $v_{\phi}=\frac{\sqrt{2}}{2} \sqrt{r} U_{g}+\frac{7}{4} U_{l}$ and $v_{g}=\sqrt{2} \sqrt{r} U_{g}+\frac{5}{2} U_{l}$

These expressions agree very well with the numerical solutions given when the full dispersion relation is solved, including for spatial solutions. Note that the $M^{-1 / 2}$ behaviour is equivalent to a linear dependence on $\alpha_{l}$, which is what was evidenced on Fig. 4. More precisely, Fig. 6 shows how the analytical expression for the frequency compares to the solution to the full dispersion relation, when the liquid to gas

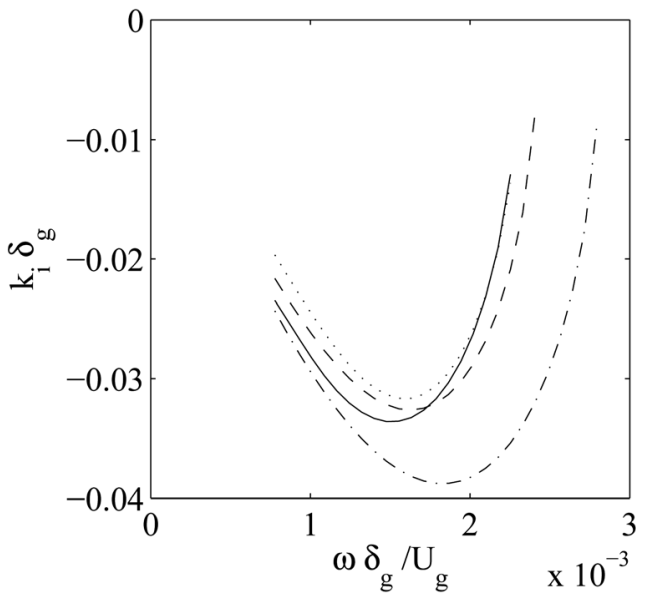

FIG. 8. Growth rate as a function of frequency, for different thicknesses of the gas stream $H_{g}$ : solid line $H_{g} / \delta_{g}=125$; dotted line $H_{g} / \delta_{g}=25$; dashed line $H_{g} / \delta_{g}=10$; dash-dotted line $H_{g} / \delta_{g}=2.5$; other parameters are $r=10^{-3}$, $\alpha_{l}=\alpha_{i}=10^{-2}$, and $n=1$.

velocity ratio $\alpha_{l}$ is varied in the range $10^{-3}-10^{-1}$. The solid line is the same curve shown on Fig. 4, and the dotted line corresponds to the expression derived above. It can be seen that the agreement is rather good as long as $\alpha_{l}<0.04$. This limit corresponds to $M>1$, which is precisely the condition on the liquid velocity needed to derive the analytical expression.

The expressions given in this section correspond to a temporal instability: it would be tempting to deduce from them a similar asymptotic expression for the spatial growth rate $k_{i}$, from the temporal growth rate $\omega_{i}$ and the group velocity based on the Gaster's relation ${ }^{18}: k_{i}=\omega_{i} / v_{g}$. However, the expression found for $k_{i}$ by this method turns out to be quite different from the expression numerically deduced from the dispersion relation. This is because Gaster's criterion for this equivalence, namely $\omega_{i} \ll \omega_{r}$, is not met here: it is incompatible with the hypothesis that $M$ is larger than one.

\section{Effect of a finite gas thickness}

We now modify the base flow (see Fig. 7) in order to take into account the finite thickness $H_{g}$ of the gas stream.

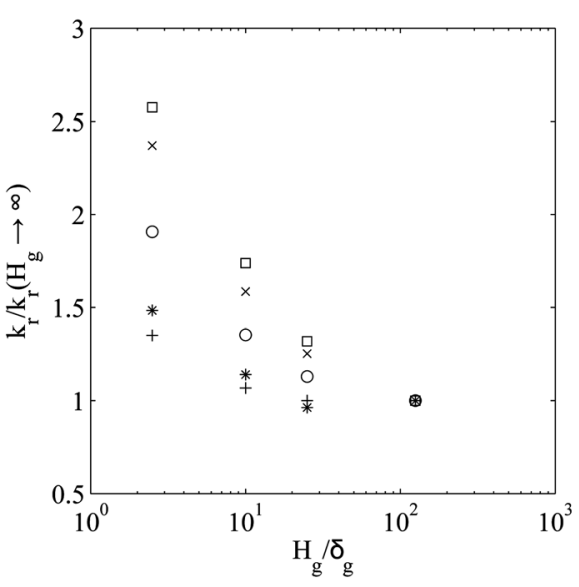

(a)

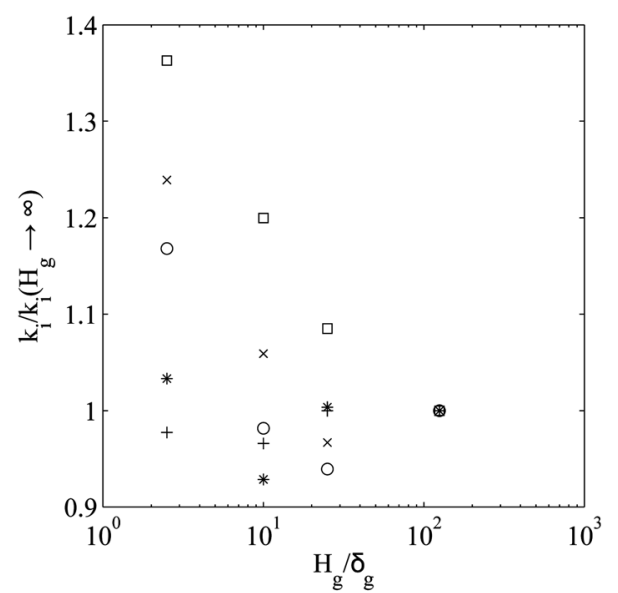

(b)
FIG. 9. Variation of the (a) real part and (b) imaginary part of the wavenumber of the most unstable mode as a function of $H_{g} / \delta_{g}$, for different density ratio $r ; \square: r=10^{-4} ; \times: r=3.10^{-4}$; $\mathrm{o:} r=10^{-3} ; *: r=5 \cdot 10^{-3} ;+: r=10^{-2}$. Other parameters are $\alpha_{l}=\alpha_{i}=10^{-2}$ and $n=1$. 


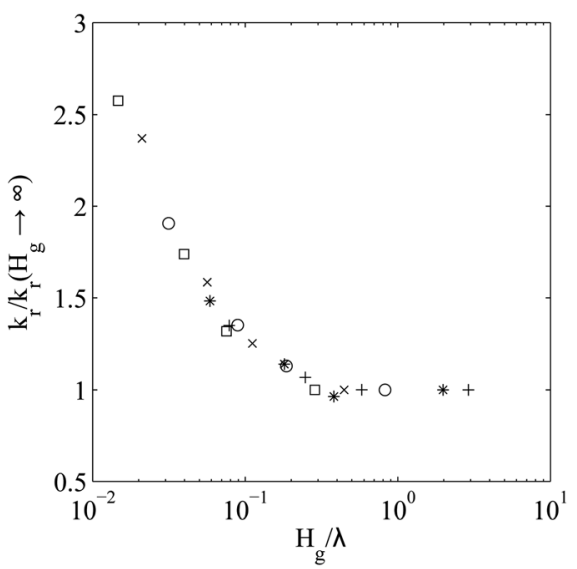

(a)

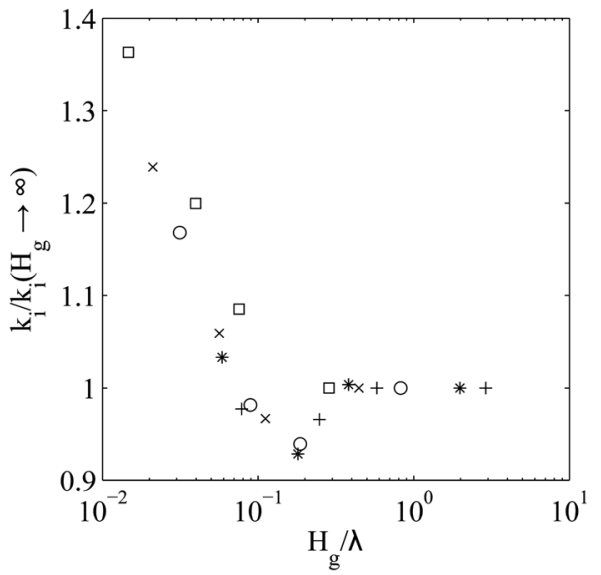

(b)
FIG. 10. Variation of the (a) real part and (b) imaginary part of the wavenumber of the most unstable mode as a function of $H_{g} / \lambda$, for different density ratio $r ; \square: r=10^{-4} ; \times: r=3.10^{-4}$; o: $r=10^{-3} ; *: r=5 \cdot 10^{-3} ;+: r=10^{-2}$. Other parameters are $\alpha_{l}=\alpha_{i}=10^{-2}$ and $n=1$.
We introduce $\delta_{g e}$, the thickness of the outer vorticity layer. The linear stability analysis is then carried out similarly as before: solutions are found in the liquid, inner vorticity layer, gas stream, outer vorticity layer, and outer region (where there is no gas flow). These solutions are matched at the boundaries, and the resulting dispersion relation is solved for spatial solutions. It can be seen on Fig. 8 that when $H_{g}$ is decreased, the growth rate is increased, and the frequency is increased. The effect of the finite thickness appears below a threshold value: on the results of Fig. 8, which were obtained for a density ratio $r=10^{-3}$ the influence of $H_{g}$ becomes relevant for $H_{g} / \delta_{g}<10$. In order to investigate if $H_{g} / \delta_{g}$ is the correct dimensionless parameter controlling this effect, we have varied the density ratio $r$ in the range $10^{-4}-10^{-2}$ : this allows to vary the wavelength of the instability independent of the vorticity thickness. Figure 9 shows that for different ratios $r$, the threshold ratio $H_{g} / \delta_{g}$ below which the instability is affected is not constant: the threshold ranges from $H_{g} / \delta_{g} \sim 50$ for $r=10^{-4}$, to $H_{g} / \delta_{g} \sim 5$ for $r=10^{-2}$. Our hypothesis is that the relevant lengthscale is the wavelength of the instability $\lambda$, which also controls the extension of the velocity perturbation in the gas phase (since the velocity perturbation is of the form $w \sim e^{-k z}$ ). We show on Fig. 10 that the ratios $k_{i} / k_{i\left(H_{g} \rightarrow \infty\right)}$ and $k_{r} / k_{r\left(H_{g} \rightarrow \infty\right)}$, respectively, collapse on a single curve when plotted as a function of $H_{g} / \lambda$. The values $k_{i\left(H_{g} \rightarrow \infty\right)}$ and $k_{r\left(H_{g} \rightarrow \infty\right)}$ are, respectively the imaginary and real part of the wavenumber in the limit $H_{g} \rightarrow \infty$. Figure 10 shows that the thickness of the gas stream $H_{g}$ starts to affect the wavelength and growth rate of the most unstable mode when $H_{g} / \lambda<0.1$ : seen the other way round, if the wavelength is larger than $10 H_{g}$ then the wavenumber and growth rate will be larger than they would be for a gas stream of infinite thickness. The same behaviour is observed for the frequency of the most unstable mode: it increases when $H_{g}$ is decreased below $0.1 \lambda$, though the values for different density ratios are less collapsed than for the wavenumber (see Fig. 11(a). All these results were obtained for the velocity profile of Fig. 7, i.e., without a velocity deficit. If the velocity deficit introduced in Sec. II A is taken into account $\left(\alpha_{i}=0\right)$, the same collapse of the data are observed, and the same curves are obtained for the wavenumber and frequency. Only the growth rate displays a slightly different behaviour, with a larger $k_{i} / k_{i\left(H_{g} \rightarrow \infty\right)}$ at small $H_{g} / \lambda$ (compare Figs. 10(b) and 11(b)): the influence of the finite thickness on the growth rate is stronger when a velocity deficit is present. All these results were obtained for an external vorticity thickness $\delta_{g e}$ equal to the inner vorticity thickness $\delta_{g}$. If $\delta_{g e}$ is varied, the same frequencies and growth rates are obtained: this parameter has no influence on the unstable modes.

In our experimental case $H_{g}$ was kept constant equal to $1 \mathrm{~cm}$, which is also the typical order of magnitude of the

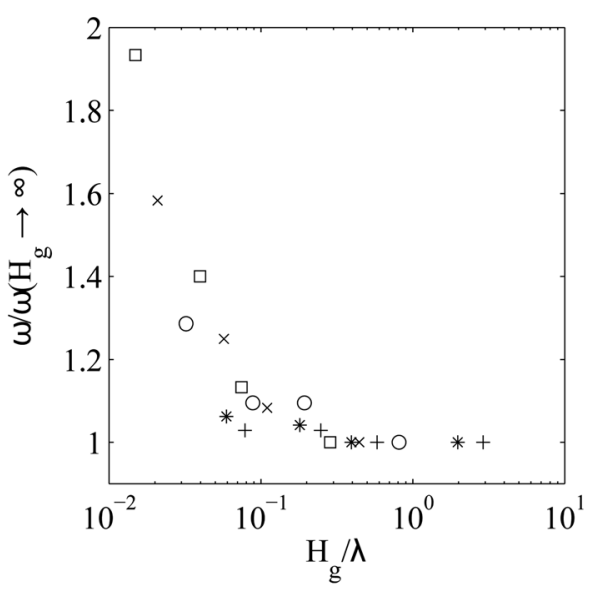

(a)

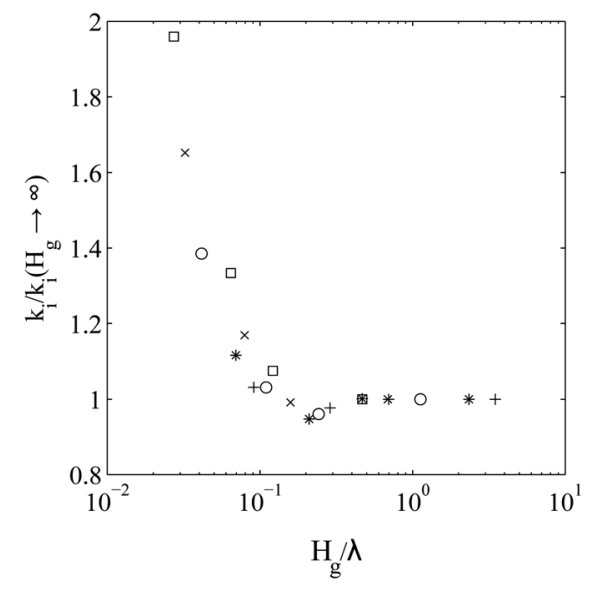

(b)
FIG. 11. (a) Variation of the frequency of the most unstable mode with $H_{g} / \lambda$, for different density ratio $r ; \square: r=10^{-4} ; \times: r=3.10^{-4} ; \mathrm{o}$ : $r=10^{-3} ; *: r=5.10^{-3} ;+: r=10^{-2}$. Other parameters are $\alpha_{l}=\alpha_{i}=10^{-2}$ and $n=1$. (b) Variation of the growth rate with $H_{g} / \lambda$, same parameters except $\alpha_{i}=0$. 


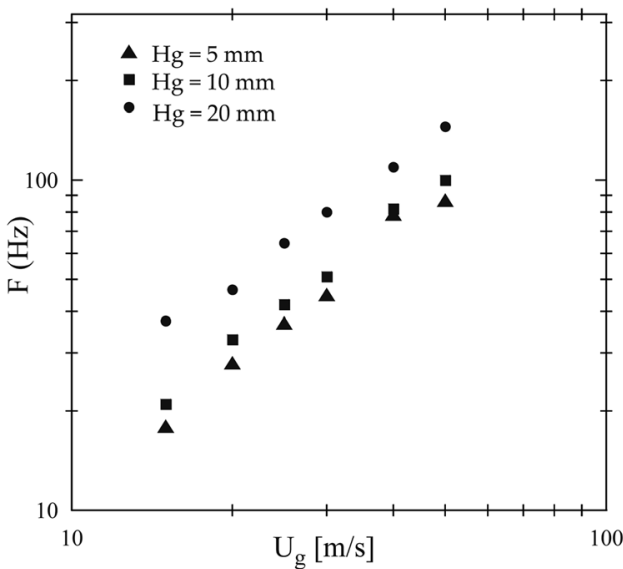

(a)

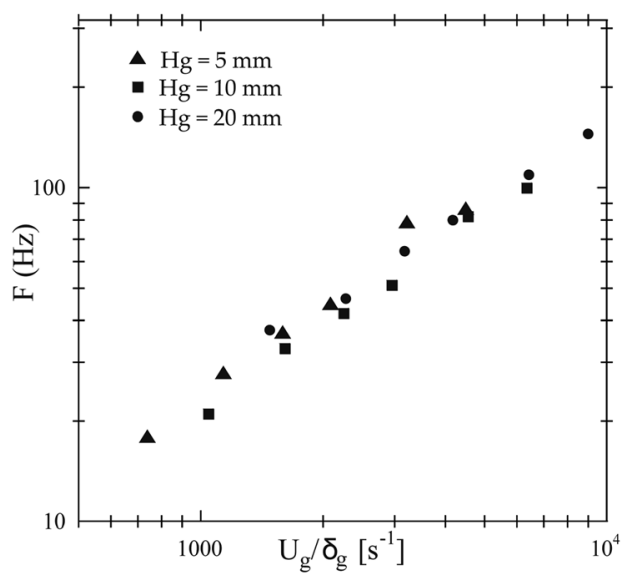

(b)
FIG. 12. Measurements of Ben Rayana9: (a) frequency of the KelvinHelmholtz instability as a function of the gas velocity, for different thicknesses $H_{g}$. (b) Same data plotted as a function of $U_{g} / \delta_{g}$ : the series are collapsed. wavelength: $H_{g}$ is therefore not expected to modify the frequency. In the coaxial set-up of Marmottant and Villermaux, ${ }^{8}$ where $H_{g}=1.7 \mathrm{~mm}$ which is smaller than the typical $\lambda$ (of the order of a centimeter), it should on the contrary affect the value predicted by the linear stability analysis. This effect might explain why their experimental growth rates were significantly larger than the ones observed on planar experiments. We will not address this coaxial geometry here, but will do so in a future study including also the effect of the cylindrical geometry on the instability.

Ben Rayana ${ }^{9,13}$ carried out frequency measurements on the same planar set-up used for this study (this set-up will be described in Sec. III, see Fig. 13) for different values of $H_{g}$, the corresponding data are shown on Fig. 12. It can be seen that the data for the three different $H_{g}$ investigated are collapsed on the same curve when plotted as a function of $U_{c} / \delta_{g}$ (Fig. 12(b). This indicates that $H_{g}$ does not modify the frequency of the unstable mode, other than via the vorticity thickness $\delta_{g}$. For the data of Fig. 12, $\lambda$ varies in the range $1.2 \mathrm{~cm}-2.5 \mathrm{~cm}$ (Ben Rayana), and three values of $H_{g}$ were investigated: $H_{g}=5 \mathrm{~mm}, H_{g}=10 \mathrm{~mm}$, and $H_{g}=20 \mathrm{~mm}$. This makes for a ratio $H_{g} / \lambda$ in the range $0.2-1.7$, i.e., above the threshold $H_{g} / \lambda=0.1$ identified in the analysis of Sec. II C.

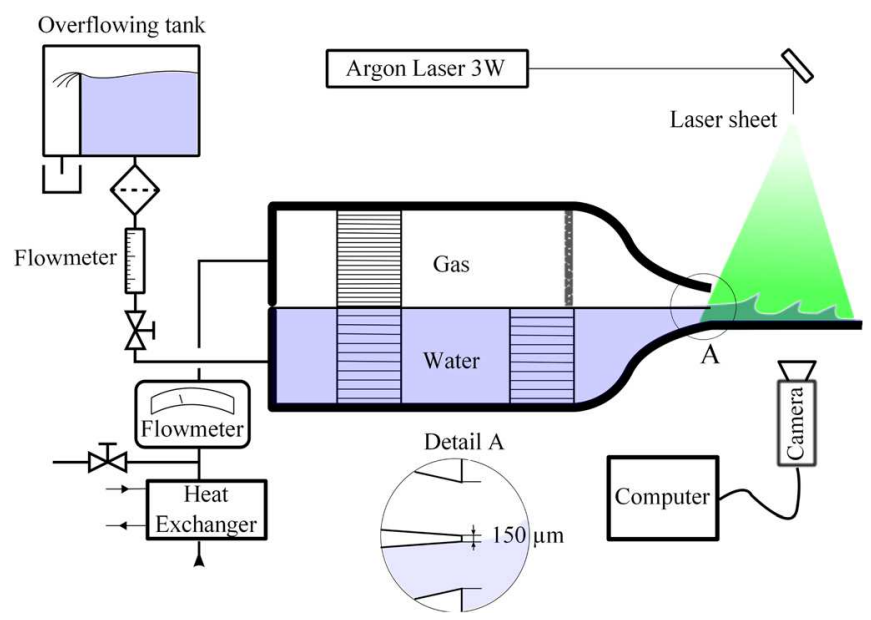

FIG. 13. (Color online) Sketch of the experimental set-up.

\section{EXPERIMENTAL RESULTS}

Our experimental set-up is a mixing layer experiment: a liquid sheet of water (width $10 \mathrm{~cm}$, thickness $H_{l}=1 \mathrm{~cm}$ ) is destabilized by a parallel gas flow of air (width $10 \mathrm{~cm}$, thickness $H_{g}=1 \mathrm{~cm}$ ). For the conditions considered here, gas velocity is in the range $U_{g}=10-30 \mathrm{~m} / \mathrm{s}$ and liquid velocity $U_{l}=0.1-1 \mathrm{~m} / \mathrm{s}$. In order to ensure a steady liquid flow, liquid is injected from an overflowing reservoir located above the experiment, with gravity driven flow. The air flow goes through a honeycomb, a porous plate and a convergent, and the liquid flow through two honeycomb plates and a symmetric convergent, all aimed at reducing velocity perturbations in each flow (see Fig. 13). The gas vorticity thickness $\delta_{g}=\Delta U /\left.\frac{d U}{d z}\right|_{\max }$ has been measured with a hot-wire anemometer, and was found to vary as $\delta_{g}=6 H_{g} R e^{-1 / 2}$ for the conditions of our experiment ${ }^{12,13}$, where $R e=U_{g} H_{g} / \nu$ with $\nu$ the kinematic gas viscosity. A small amount of fluoresceine is added in the liquid phase, we make a longitudinal laser sheet (Argon laser) of the liquid flow, and a fast camera (Phantom v12) records the section of the jet. Hence, the location of the interface can be obtained by image processing as a function of the downstream position, and of time. The height of the interface at a given downstream position is then Fourier transformed using the Welch's method with MATLAB. If the number of images is large enough (typically several hundred periods), a peak is observed to dominate the noise in the spectrum. Its maximum is then identified as the frequency of the instability. Figure 14(a) shows two typical spectra obtained by this method: depending on the gas/liquid velocities the aspect ratio of the maximum peak can vary. For given flow conditions, this spectrum can be made at different downstream locations: Fig. 14(b) shows spectra computed every $2 \delta_{g}$, up to a downstream position $x=65 \delta_{g}$ ( $\mathrm{x}$ is counted from the injector). The amplitude of the maximum of the spectrum increases with downstream location. This is due to the increasing amplitude of the waves, as the liquid interface goes from undergoing a slight oscillation to being atomized. Note that though the value of the maximum peak is modified, the corresponding frequency remains unchanged. Beyond $x=65 \delta_{g}$ the location of the highest amplitude spectrum shown of Fig. 14(b), the amplitude decreases, but again the frequency of the maximum remains 


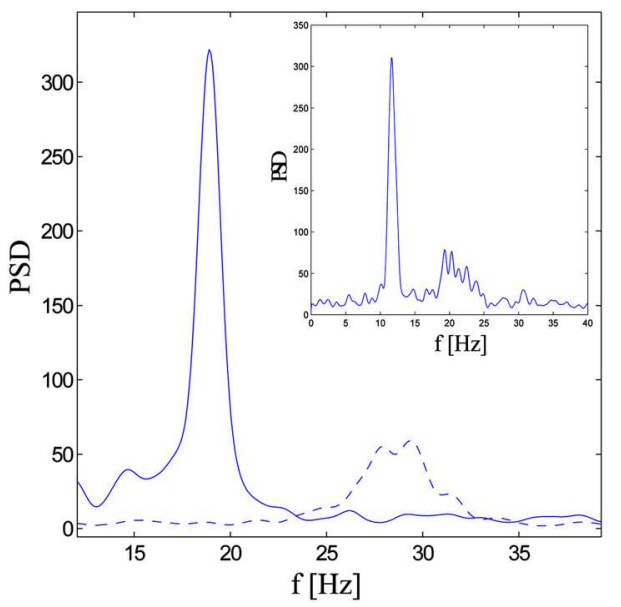

(a)

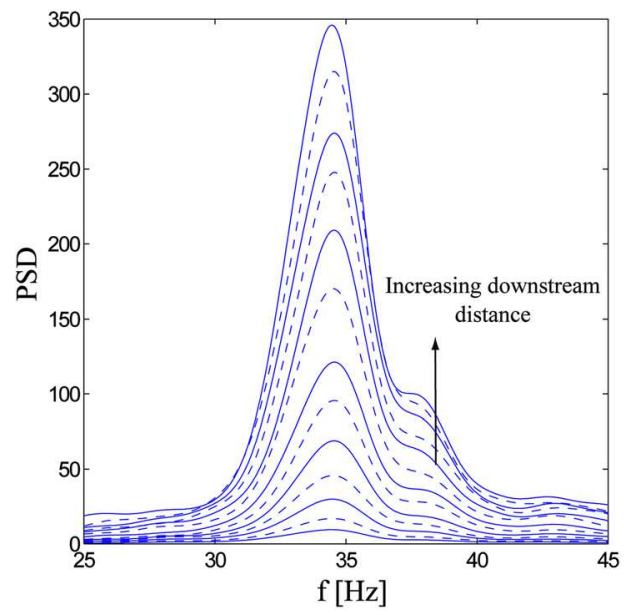

(b)
FIG. 14. (Color online) (a) Example of a spectrum of the position of the interface, for $U_{g}=22 \mathrm{~m} \mathrm{~s}^{-1}$ : solid line $U_{l}=0.26 \quad \mathrm{~m} \quad \mathrm{~s}^{-1}$, dashed line $U_{l}=0.5 \mathrm{~m} \mathrm{~s}^{-1}$. The insert, on a larger scale, shows harmonics for $U_{g}=12 \mathrm{~m} \mathrm{~s}^{-1}$ and $U_{l}=0.26 \mathrm{~m} \mathrm{~s}^{-1}$; (b) Downstream variation of the spectrum of the amplitude of the instability: $U_{g}=12 \mathrm{~m} \mathrm{~s}^{-1}$, $U_{l}=0.26 \mathrm{~m} \mathrm{~s}^{-1}$. The spectrum is computed every $2 \delta_{g}$, up to $x=65 \delta_{g}$ : the amplitude of the maximum increases with downstream distance. the same. This is different from the behaviour observed by Fuster et $a .^{19}$ on their numerical simulation of a similar liquid-gas configuration, where the frequency decreases with the downstream distance.

This frequency is measured for various liquid and gas velocities. Below the smallest gas velocity investigated $\left(U_{g}=12 \mathrm{~m} \mathrm{~s}^{-1}\right)$ the amplitude of the perturbation is too small given our optical resolution. Beyond $U_{g}=30 \mathrm{~m} \mathrm{~s}^{-1}$, measurement is made difficult by the break-up of the waves; in addition, we took care to measure the growth rate of the instability conjointly with the frequency for each set of conditions (the method will be detailed below), and for $U_{g}>30 \mathrm{~m} \mathrm{~s}^{-1}$ the rapid saturation of the waves impairs the measurement of the growth rate. Figure 15 shows that the frequency increases with both the gas velocity and the liquid velocity.

The analysis of Sec. II has predicted a dimensionless frequency varying with $M^{-1 / 2}$, if $M$ is not small. We therefore plot on Fig. 16 the variations of $f \delta_{g} / U_{g}$ as a function of $M^{-1 / 2}$ : the data of Fig. 15 collapse on a single curve. The dotted line shows the prediction derived for $M>1$ for the velocity profile of Fig. 1(b), while the solid line represents the prediction when a full velocity deficit is taken into account

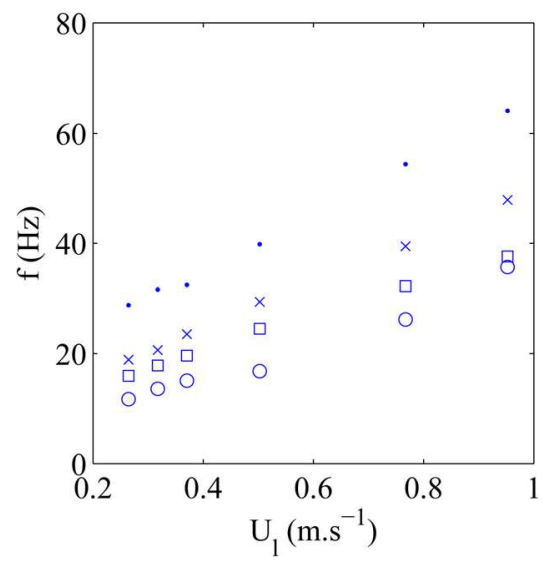

FIG. 15. (Color online) Experimental frequency as a function of the liquid velocity $U_{l}$, for different $U_{g}: \bigcirc: U_{g}=12 \mathrm{~m} \mathrm{~s}^{-1} ; \square: U_{g}=17 \mathrm{~m} \mathrm{~s}^{-1} ; \times$ : $U_{g}=22 \mathrm{~m} \mathrm{~s}^{-1} ; \bullet: U_{g}=27 \mathrm{~m} \mathrm{~s}^{-1}$. $\left(\alpha_{i}=0\right)$. It can be seen that the agreement with the velocity deficit prediction is very good. We now compare on Fig. 17 our experimental results to the experimental results of Raynal et al. ${ }^{7}$ (crosses) and Ben Rayana ${ }^{9,13}$ (squares). As mentioned in the introduction, these frequencies are both larger than the classical inviscid prediction without a velocity deficit, shown as a dotted line. Our prediction derived from the analysis with a velocity deficit (solid line) is in a rather good agreement with Raynal data, except for large values of $\mathrm{M}$. This could be due to the change in topology when $M$ becomes large: due to the strong atomization the length $L$ of the intact liquid core shortens: $L \approx 6 H_{g} / \sqrt{M}$, as proposed by Raynal. ${ }^{12}$ Hence the interface becomes steeper and the quasi parallel flow assumption is no longer valid. Ben Rayana data (obtained for a fixed $\mathrm{M}=16$ ) is also closer to our prediction than to the classical one, but it is still at about twice the predicted value. Ben Rayana data (all for $M=16$ ) were obtained by visualization, through counting of the number of waves over a given duration, while both Raynal and our method are the spectral methods, which identify the maximum of a spectrum derived from the interface location.

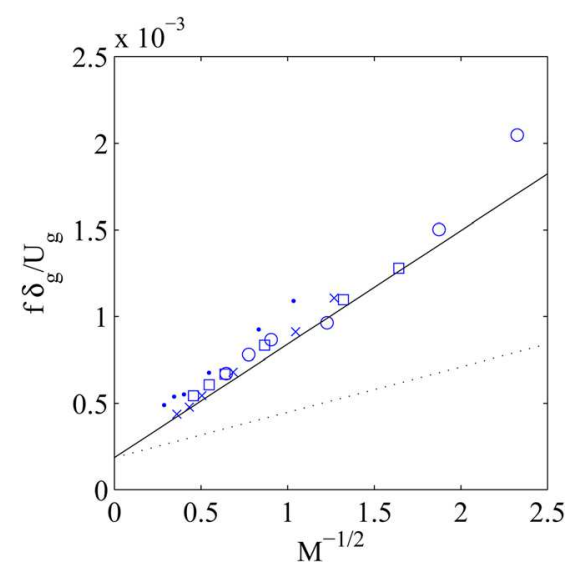

FIG. 16. (Color online) Dimensionless frequency as a function of $M^{-1 / 2}$. The dotted line is $f=(r / 2 \pi)\left(1+\sqrt{2} M^{-1 / 2}\right)$ (asymptotic prediction for profile of Fig. 1(b) and the solid line is $f=(r / 2 \pi)\left(1+\frac{5}{2} \sqrt{2} M^{-1 / 2}\right)$ (asymptotic prediction for profile of Fig. 1(c). Symbols correspond to different values of $U_{g}, \bigcirc$ : $U_{g}=12 \mathrm{~m} \mathrm{~s}^{-1} ; \square: U_{g}=17 \mathrm{~m} \mathrm{~s}^{-1} ; \times: U_{g}=22 \mathrm{~m} \mathrm{~s}^{-1} ; \bullet: U_{g}=27 \mathrm{~m} \mathrm{~s}^{-1}$ 


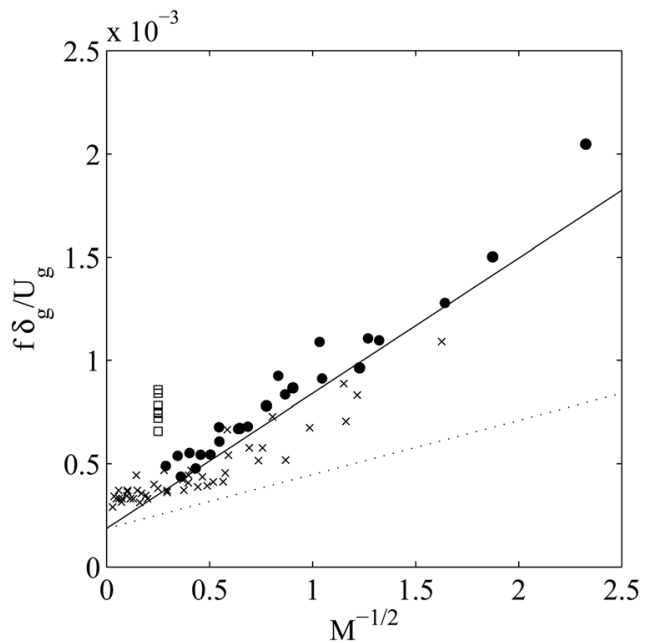

FIG. 17. Comparison of data sets for the dimensionless frequency as a function of $M^{-1 / 2}$ : x results of Raynal ${ }^{7,12} ; \square$ results of Ben Rayana ${ }^{9,13}$; $\bullet$ results of the present study. The dotted line is $f=(r / 2 \pi)\left(1+\sqrt{2} M^{-1 / 2}\right)$ (asymptotic prediction for profile of Fig. 1(b) and the solid line is $f=(r / 2 \pi)\left(1+\frac{5}{2} \sqrt{2} M^{-1 / 2}\right)$ (asymptotic prediction for profile of Fig. 1(c).

Spectra for large $\mathrm{M}$ are usually quite noisy and display strong harmonics: this may explain why the frequency measured by Ben Rayana et al. ${ }^{9}$ as an average frequency is significantly larger than the maximum frequency retained by spectral methods.

In order to be more precise on the comparison of the linear stability analysis frequency and the experimental frequency, we plot on Fig. 18(a) the ratio of the experimental to predicted frequencies, as a function of $\mathrm{M}$. The predicted frequency is here the solution of the spatial inviscid stability analysis, taking into account a full velocity deficit in the liquid phase, as well as gravity and surface tension. Though this ratio is in average close to one (which is not the case if the velocity deficit is not taken into account, as evidenced by Fig. 16), the points are quite scattered: the ratio varies between 0.8 and 1.2 for the conditions of our present experiment. Note that for low $\mathrm{M}$ the theory tends to overestimate the experimental frequency (ratio $f_{\text {exp }} / f_{\text {th }}$ as low as 0.8 ), a behaviour not observed on Fig. 16 where the experimental frequency was compared to the asymptotic prediction: this difference is expected since the asymptotic law of section B departs from the full solution precisely for low M, i.e. large $\alpha_{l}$ (Fig. 6), but what is unexpected is that the experimental points end up closer to the asymptotic law than to the exact numerical solution of the dispersion relation. Figure 18(b) next shows the same ratio $f_{\text {exp }} / f_{\text {th }}$ computed for the experimental points of Raynal et al. ${ }^{7}$ and Ben Rayana. ${ }^{9}$ The data points of Raynal et al. (crosses) lie along a curve going from a ratio $f_{\text {exp }} / f_{\text {th }}=0.7$ to a ratio 2 , across the several orders of magnitude of $\mathrm{M}$ spanned by their data. Though for a given value of $\mathrm{M}$ there is a strong dispersion in the frequency ratio, this global trend suggests that $\mathrm{M}$ has a strong influence on the departure to the prediction of the linear stability analysis. As mentioned in the discussion of Fig. 17, this could be due to the parallel flow assumption not being valid at large $M$, in particular when the liquid intact length $L$ becomes shorter than the wavelength. The same ratio $f_{\text {exp }} / f_{\text {th }}$ is shown for the data of Ben Rayana, all for a fixed $M=16$ : the ratio of frequencies is larger for these measurements than for other data sets, in line with the difference observed on Fig. 17. As mentioned in the preceding paragraph, this could be due to a bias introduced by the counting method used by Ben Rayana.

We also carried out measurements of the spatial growth rate $k_{i}$ of the instability, using the following method: we superpose each captured interface; for a given downstream location, we make a histogram of the interface positions (typically over several hundred periods of the instability), and exclude the lowest and highest $1 \%$ events: this procedure aims at removing single events (a much larger isolated wave for example) which might occur over the course of the measurement. The remaining width of the histogram is then taken to be the amplitude $A$ of the waves. Figure 19(a) illustrates this procedure: the solid line shows the position of the interface for a given time $t$; the histograms below were made at the locations shown by the three dot dashed lines; the dashed lines on the histograms show the limit value retained for the amplitude after the exclusion of the lowest and highest $1 \%$; the dashed line wrapping the interface corresponds to this same limits once reported on the mixing layer. The amplitude is plotted as a function of downstream position, for a fixed $U_{l}=0.37 \mathrm{~m} \mathrm{~s}^{-1}$ and for the different $U_{g}$ investigated, see Fig. 19(b). It can be seen that there is a region of exponential growth (enhanced by the dashed line on the graph), whose extent decreases when $U_{g}$ is increased: after a limit position, the growth becomes slower, indicating a

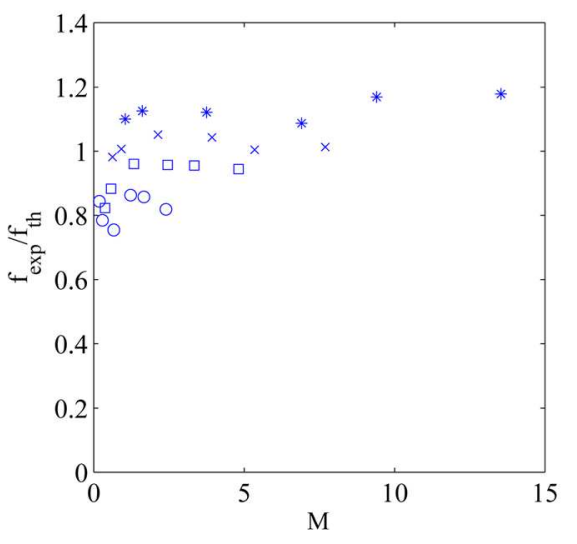

(a)

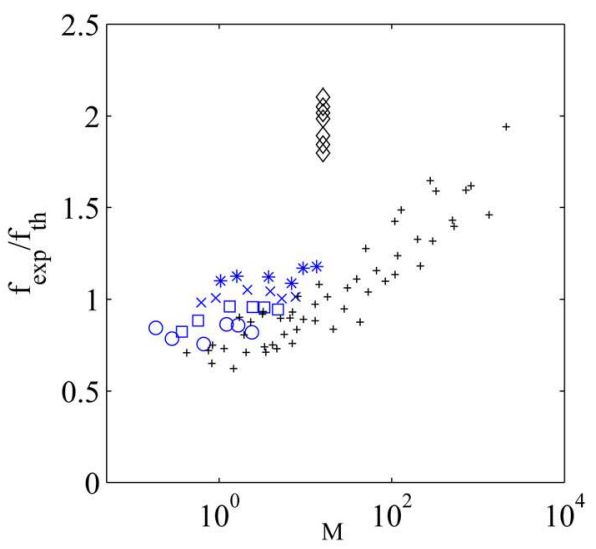

(b)
FIG. 18. (Color online) (a) Ratio of the experimental frequency and the predicted frequency, as a function of M. Symbols correspond to different values of $U_{g}, \bigcirc: U_{g}=12 \mathrm{~m} \mathrm{~s}^{-1}$; $\square: U_{g}=17 \mathrm{~m} \mathrm{~s}^{-1} ; \times: U_{g}=22 \mathrm{~m} \mathrm{~s}^{-1} ; *$ : $U_{g}=27 \mathrm{~m} \mathrm{~s}^{-1}$. (b) Same plot with the data of Raynal (crosses) and Ben Rayana (diamonds). 


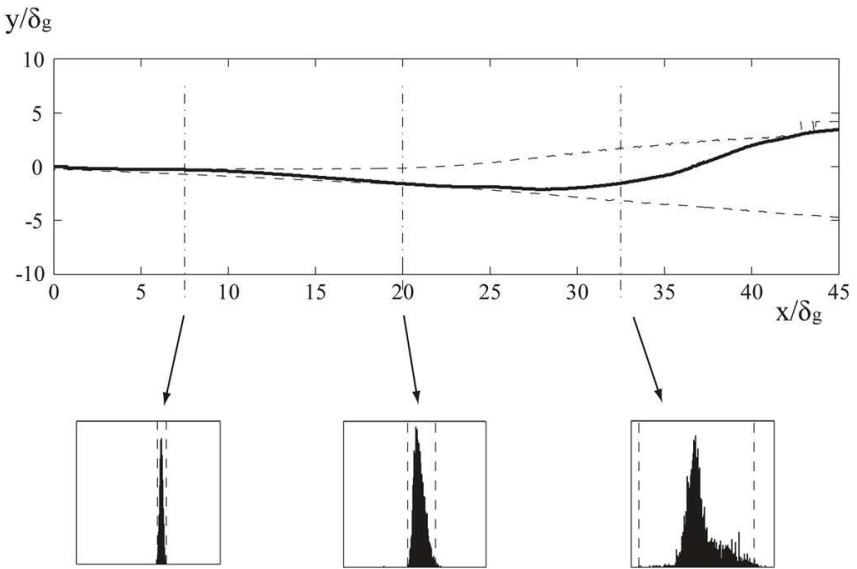

(a)

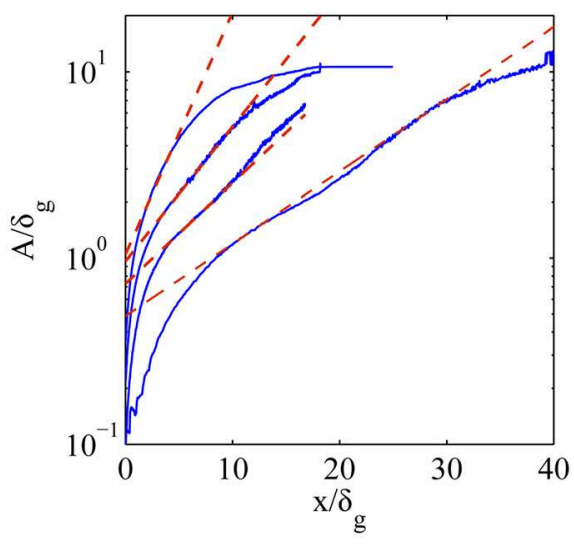

(b)

FIG. 19. (Color online) (a) Illustration of the growth rate measurement $\left(U_{g}=12 \mathrm{~m} / \mathrm{s}, U_{l}=0.37 \mathrm{~m} / \mathrm{s}\right)$ : a histogram of the interface positions at a given downstream position is made; the amplitude of the instability is deduced from the width of the histogram (dashed line); (b)Variation of the dimensionless amplitude $A / \delta_{g}$ of the waves, as a function of downstream distance. From right to left, $U_{g}=12,17,22$, and $27 \mathrm{~m} \mathrm{~s}^{-1}$. The region of exponential growth (enhanced by the dashed line) is drastically reduced as $U_{g}$ is increased.

possible saturation. It is interesting to note that the region of exponential growth is preceded by another region where the growth rate steadily decreases: the extent of this latter region decreases when $U_{g}$ is increased. For each gas velocity, the region of exponential growth appears to be reached when the amplitude of the waves reaches a threshold value of approximately $0.7 \delta_{g}$. The nature of this first region remains unclear: our guess is that it could be due to a wake caused by the splitter plate, and at any rate be caused by the finite thickness of this plate ( $e=150 \mu \mathrm{m}$ at its end).

We now present on Fig. 20 the values of the dimensionless spatial growth rate of the instability measured in the zone of exponential growth, as a function of the gas velocity $U_{g}$ and as a function of M. It can be seen that $k_{i}$ increases steadily with $U_{g}$, (Fig. 20(b)) while it is extremely scattered when plotted as a function of M (Fig. 20(a)). This indicates that while $\mathrm{M}$ controls the variations of the dimensionless frequency, it does not control the variations of the dimensionless spatial growth rate. The latter increases roughly as $U_{g}^{2}$. This experimental growth rate can be compared to the spatial

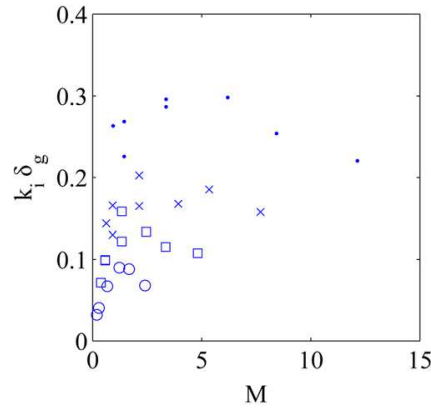

(a)

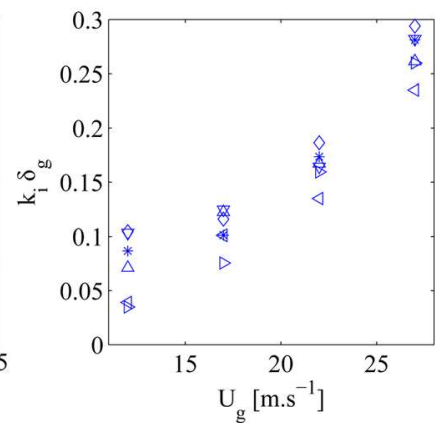

(b)
FIG. 20. (Color online) (a) Dimensionless measured growth rate as a function of $\mathrm{M},{ }^{\circ}: U_{g}=12 \mathrm{~m} \mathrm{~s}^{-1} ; \square: U_{g}=17 \mathrm{~m} \mathrm{~s}^{-1}$; $\mathrm{x}: U_{g}=22 \mathrm{~m} \mathrm{~s}^{-1}$; • $U_{g}=27 \mathrm{~m} \mathrm{~s}^{-1}$; (b) Dimensionless measured growth rate as a function of $U_{g}$; *: $U_{l}=0.26 \mathrm{~m} \mathrm{~s}^{-1} ; \diamond: U_{l}=0.31 \mathrm{~m} \mathrm{~s}^{-1} ; \nabla: U_{l}=0.37 \mathrm{~m} \mathrm{~s}^{-1} ; \triangle:$ $U_{l}=0.50 \mathrm{~m} \mathrm{~s}^{-1} ; \triangleleft: U_{l}=0.76 \mathrm{~m} \mathrm{~s}^{-1} ; \triangleright: U_{l}=0.95 \mathrm{~m} \mathrm{~s}^{-1}$. growth rate $k_{i t h}$ deduced from the spatial stability analysis (numerical resolution of the dispersion relation, with a full velocity deficit, gravity perpendicular to the interface and surface tension included). Figure 21 shows the variations of the ratio $k_{\text {iexp }} / k_{\text {ith }}$ as a function of the gas velocity. It can be seen that this ratio is around two for the lowest gas velocity investigated, but when $U_{g}$ is increased it increases up to a ratio eight between the experimental and predicted growth rate. These variations are quite similar to the variations of the experimental $k_{i}$ shown on Fig. 20(b): this is because the gas velocity has very little influence on the spatial growth rate predicted by the stability analysis. Figure 21 shows that the measured growth rate is much larger than the predicted one: while it manages to capture the frequency of the instability, the inviscid stability analysis strongly underestimates its growth rate. This could be due to the strong spatial variations induced by the instability: PIV measurements of the gas velocity field carried out on an analogous but axisymmetric coaxial jet set-up showed that when the amplitude of

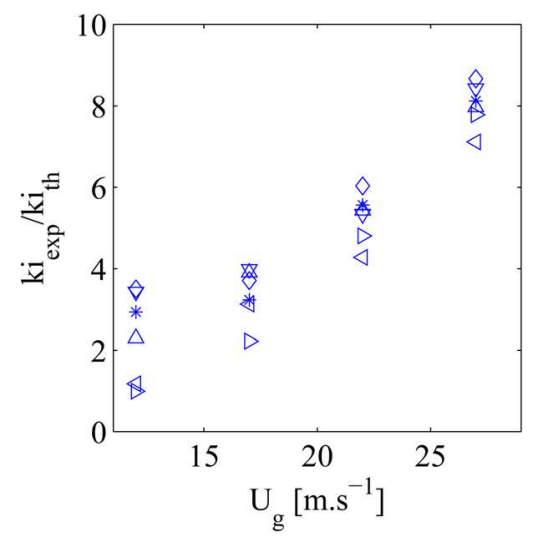

FIG. 21. (Color online) Ratio of the experimental and predicted spatial growth rate, as a function of $U_{g}$. Symbols correspond to different values of $U_{l}, *: U_{l}=0.26 \mathrm{~m} \mathrm{~s}^{-1} ; \diamond: U_{l}=0.31 \mathrm{~m} \mathrm{~s}^{-1} ; \nabla: U_{l}=0.37 \mathrm{~m} \mathrm{~s}^{-1} ; \triangle:$ $U_{l}=0.50 \mathrm{~m} \mathrm{~s}^{-1} ; \triangleleft: U_{l}=0.76 \mathrm{~m} \mathrm{~s}^{-1} ; \triangleright: U_{l}=0.95 \mathrm{~m} \mathrm{~s}^{-1}$. 

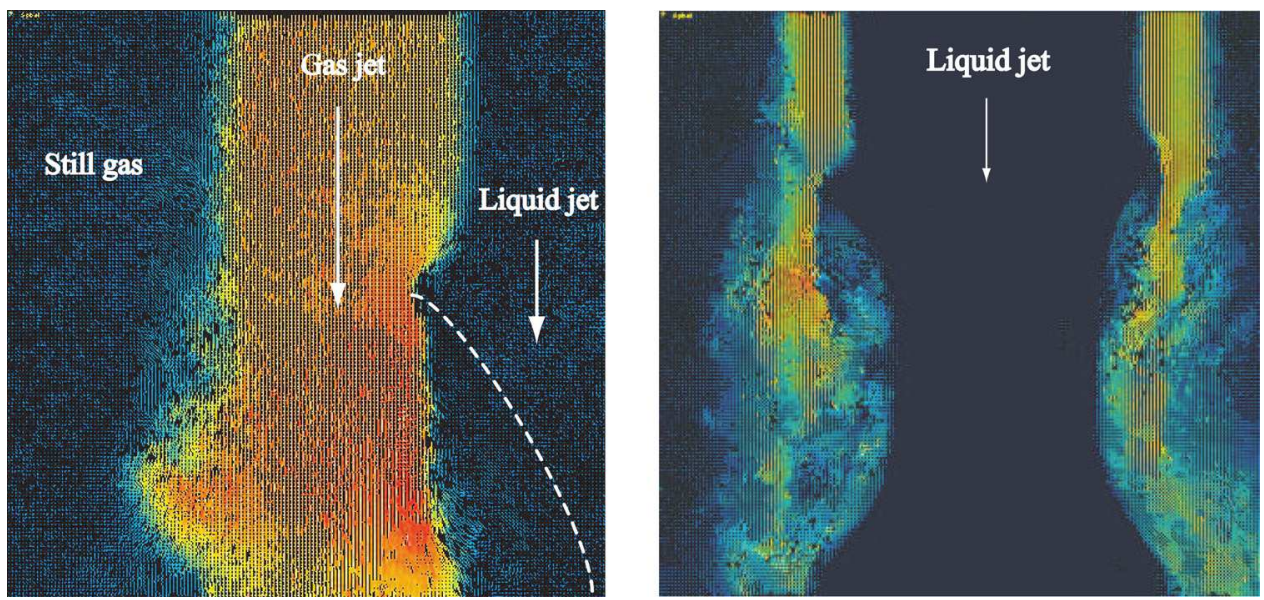

FIG. 22. (Color online) PIV visualization of the gas flow around a wave, for an annular gas flow $\left(H_{g}=1.5 \mathrm{~mm}\right)$ around a liquid jet (radius $R=4 \mathrm{~mm}$ ), $U_{l}=0.5 \mathrm{~m} \mathrm{~s}^{-1}, U_{\mathrm{g}}=20 \mathrm{~m} \mathrm{~s}^{-1}$. The white dashed line enhances the limit of the liquid jet downstream the wave: the gas jet is detached from the liquid. the waves exceeds $\delta_{g}$, the vorticity thickness becomes significantly reduced at the crest of the waves: downstream the crest the gas jet then detaches from the liquid, creating low velocity recirculations in the following trough of the wave. A typical velocity field obtained on this coaxial set-up is shown on Fig. 22. The variations of the amplitude of the waves shown on Fig. 19(b) show that the distance after which the amplitude reaches $\delta_{g}$ varies from $x \sim 10 \delta_{g}$ for $U_{g}=12 \mathrm{~m} \mathrm{~s}^{-1}$, to $x \sim \delta_{g}$ for $U_{g}=27 \mathrm{~m} \mathrm{~s}^{-1}$ : the typical picture of Fig. 22 is therefore expected to occur well before the end of the first wavelength, even for relatively low gas velocities. These strong spatial variations in the experiment are likely to play a part in the departure from the predictions of the stability analysis.

Following the idea that the departure from the growth rate predicted by the linear stability analysis is caused by the large amplitude of the waves, we plot on Fig. 23 the ratio of the measured to predicted frequency, as a function of the experimental spatial growth rate. The predicted frequency, as in Fig. 18, is the numerical solution of the dispersion relation including surface tension, gravity and a full velocity deficit $\left(\alpha_{i}=0\right)$. It can be seen that the data points of Fig. 18(a) are now significantly less scattered: this is because among a series corresponding to a given $U_{g}$ (given symbol), an increasing $k_{i}$ corresponds in average to an increasing $f_{\text {exp }} / f_{t h}$. The fact that for low growth rates the ratio $f_{\text {exp }} / f_{\text {th }}$ is smaller than one (as low as 0.8 ) could be due to an overestimation of the

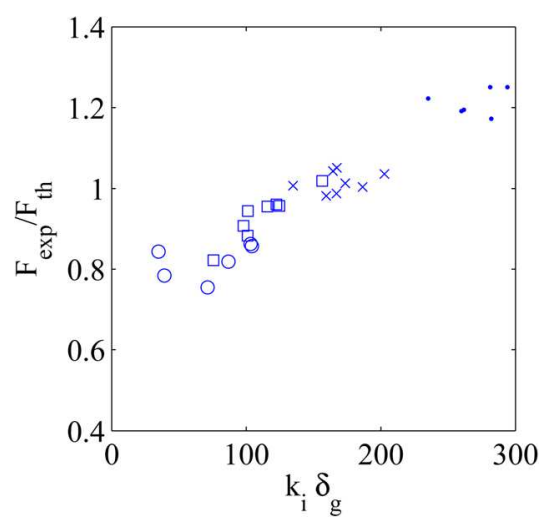

FIG. 23. (Color online) Ratio of the experimental and predicted frequency as a function of the experimental growth rate. velocity deficit: a zero velocity at the interface has been assumed in the stability results presented here. When the experimental growth rate of the instability becomes larger it causes steeper spatial variations, accelerating the failure of the stability analysis: the ratio $f_{\text {exp }} / f_{\text {th }}$ increases, and the experimental growth rate strongly departs from its prediction.

\section{CONCLUSION}

We have extended the inviscid stability analysis of a liquid stream destabilized by a parallel gas stream, in order to take into account key features of the velocity profile: namely the liquid velocity deficit caused by the splitter plate at the liquid-gas interface, and the finite thickness of the gas stream. We find that the velocity deficit leads to a significant increase in the predicted frequency of the most unstable mode. It also predicts a stronger influence of the liquid velocity on this frequency, and it removes the inviscid liquid mode (scaling with $\delta_{l}$ ) not observed in the experiments but predicted by previous analyses. ${ }^{3}$ The effect of a finite gas stream $H_{g}$ is also to increase the frequency of the instability: this effect becomes relevant for $H_{g}<0.1 \lambda$. Note however that the scaling of the most unstable mode remains essentially controlled by the vorticity thickness of the fast gas phase.

We have carried out experimental measurements of the frequency and of the growth rate of the instability. The results show a good agreement of the measured frequency with the frequency predicted by the inviscid stability analysis, provided that the liquid velocity deficit at the interface is taken into account. The experimental growth rate is on the contrary significantly larger than the predicted growth rate. We attribute this to the strong spatial variations observed in the experiment, and in particular to the impact of the large amplitude of the waves on the gas flow.

The present analysis is inviscid: recent studies including viscosity in the stability analysis of a similar two phase mixing layer (Boeck and Zaleski, ${ }^{15}$ Fuster et al. ${ }^{19}$ ), but starting from a smooth version of the base flow of Fig. 1(a), predict a different most unstable mode for our experimental conditions, not scaling with $\delta_{g}$ as is observed in the experiment. It would be interesting to extend these viscous analyses to a smooth version of the velocity profile of Fig. 1(c), to determine how the interface velocity affects the viscous mode, 
and to see in particular if the most unstable modes in both the viscous and inviscid cases turn out to be similar when the deficit is included.

In most industrial applications of coaxial injectors the geometry of the flow is axisymmetric, and not planar. The same inviscid mode, driven by the gas vorticity thickness, has been observed on experiments carried out in this geometry by Marmottant and Villermaux ${ }^{8}$, but with discrepancies between the predicted frequency and the experimental frequency. We believe that the inclusion of the finite thickness $H_{g}$ of the gas stream and of the cylindrical geometry in the theory could significantly affect the results of the predictions for this geometry.

${ }^{1}$ A.H. Lefebvre, Atomization and Sprays (Hemisphere, New York, 1989).

${ }^{2}$ J. Lasheras and E. J. Hopfinger, "Liquid jet instability and atomization in a coaxial gas stream," Annu. Rev. Fluid Mech. 32, 275 (2000).

${ }^{3}$ J. Eggers and E. Villermaux, "Physics of liquid jets," Rep.Prog.Phys. 71, 036601 (2008).

${ }^{4}$ L. Rayleigh, "On the stability, or instability, of certain fluid motions," Proc. London Math. Soc. 11, 57 (1879).

${ }^{5}$ G. A. Lawrence, F. K. Browand, and L. G. Redekopp, "The stability of a sheared density interface," Phys. Fluids A 3(10), 2360 (1991).

${ }^{6} \mathrm{H}$. Eroglu and N. Chigier, "Wave characteristics of liquid jets from airblast coaxial atomizers," Atomization Sprays 1, 349 (1991).

${ }^{7}$ L. Raynal, E. Villermaux, J. Lasheras, and E. J. Hopfinger, "Primary instability in liquid gas shear layers," in 11th Symposium on Turbulent Shear Flows, 7-10 September 1997, Grenoble, France, Vol. 3, pp. 27.1-27.5, OCLC 40626641, INP-CNRS-UJF.
${ }^{8}$ P. Marmottant and E. Villermaux, "On spray formation," J. Fluid. Mech. 498, 73 (2004).

${ }^{9}$ F. Ben Rayana, A. Cartellier, and E. J. Hopfinger, "Assisted atomization of a liquid layer: Investigation of the parameters affecting the mean drop size prediction," (paper ICLASS06-190), in Proceedings of the international conference on Liquid Atomization and Spray Systems (ICLASS), 27 August-1 September, Kyoto, Japan, ISBN 4-9902774-1-4 (Academic Publication and Printings Co. Ltd., Osaka, Japan, 2006).

${ }^{10}$ E. Villermaux, "Mixing and spray formation in coaxial jets," J. Propul. Power 14, 807 (1998).

${ }^{11}$ J. M. Gordillo, M. Pérez-Saborid, and A. M. Gañán-Calvo, "Linear stability of co-flowing liquid-gas jets," J. Fluid. Mech. 448, 23 (2001).

${ }^{12}$ L. Raynal, "Instabilité et entraînement à l'interface d'une couche de mélange liquide-gaz”, Ph.D. dissertation, Université J. Fourier Grenoble I, France, 1997.

${ }^{13} \mathrm{~F}$. Ben Rayana, "Contribution à l'étude des instabilités interfaciales liquide-gaz en atomisation assistée et tailles de gouttes", Ph.D. dissertation, INP Grenoble, France, 2007.

${ }^{14}$ P. E. Dimotakis, “Two-dimensional shear-layer entrainment," AIAA J. 24, 1791 (1986).

${ }^{15}$ T. Boeck and S. Zaleski, "Viscous versus inviscid instability of two-phase mixing layers with continuous velocity profile," Phys. Fluids 17, 032106 (2005).

${ }^{16} \mathrm{E}$. Hinch, "A note on the mechanism of the instability at the interface between two fluids," J. Fluid Mech. 144, 463 (1984).

${ }^{17}$ S. Chandrasekhar, Hydrodynamic and Hydromagnetic Stability (Dover, New York, 1981).

${ }^{18} \mathrm{M}$. Gaster, "A note on the relation between temporally-increasing and spatially-increasing disturbances in hydrodynamic stability," J. Fluid. Mech. 14, 222 (1962).

${ }^{19}$ D. Fuster, A. Bagué, T. Boeck, L. Le Moyne, A. Leboissetier, S. Popinet, P. Ray, T. R. Scardovelli, and S. Zaleski, "Simulation of primary atomization with an octree adaptive mesh refinement and VOF method," Int. J. Multiphase Flow 35, 550 (2009). 